\title{
Shape simplification through polygonal approximation in the Fourier domain
}

\section{Mark Andrews, Ramakrishna Kakarala}

Mark Andrews, Ramakrishna Kakarala, "Shape simplification through polygonal approximation in the Fourier domain," Proc. SPIE 9406, Intelligent Robots and Computer Vision XXXII: Algorithms and Techniques, 94060D (8 February 2015); doi: 10.1117/12.2078148

Event: SPIE/IS\&T Electronic Imaging, 2015, San Francisco, California, United States 


\title{
Shape simplification through polygonal approximation in the Fourier domain
}

\author{
Mark Andrews ${ }^{a}$ and Ramakrishna Kakarala ${ }^{b}$ \\ ${ }^{a}$ Department of Electrical and Computer Engineering, University of Auckland, New Zealand; \\ ${ }^{b}$ School of Computer Engineering, Nanyang Technological University, Singapore
}

\begin{abstract}
Fourier descriptors have long been used to describe the underling continuous contours of two-dimensional shapes. Approximations of shapes by polygons is a natural step for efficient algorithms in computer graphics and computer vision. This paper derives mathematical relationships between the Fourier descriptors of the continuous contour, and the corresponding descriptors of a polygon obtained by connecting samples on the contour. We show that the polygon's descriptors may be obtained analytically in two ways: first, by summing subsets of the contour's descriptors; and second, from the discrete Fourier transform (DFT) of the polygon's vertices. We also analyze, in the Fourier domain, shape approximation using interpolators. Our results show that polygonal approximation, with its potential benefits for efficient analysis of shape, is achievable in the Fourier descriptor domain.
\end{abstract}

Keywords: Fourier series, curves, polygons, DFT, sampling

\section{INTRODUCTION}

As many papers have demonstrated, the bounding contour of an object suffices to detect, classify, or recognize an object in an image. ${ }^{1,2}$ The contour of an object in the physical world is by nature continuous, while its appearance in an image is discretized by the pixel grid. Accurate measurement of object properties, such as enclosed area or perimeter, requires a representation of the underlying continuous bounding contour.

Fourier descriptors have long been used to represent continuous contours. ${ }^{3}$ The value in Fourier descriptors lies, in part, in the way that they allow the powerful machinery of Fourier analysis to illuminate shape transformations such as rigid transformation and simplification through filtering. Indeed, it is possible to study Fourier descriptors as a branch of applied harmonic analysis, without reference to their applications in computer vision. It is in that spirit that this paper is devoted to the analysis of polygonal approximation in the Fourier domain.

Approximating shapes with polygons is a natural method for simplifying them, and is useful for applications in computer graphics ${ }^{4}$ and computer vision. ${ }^{5}$ However, existing research does not provide any mathematical relationships between the Fourier descriptors of a continuous contour and those of a polygon obtained by connecting samples taken on the contour. In this paper, we derive the theory of approximating shapes by polygons operating entirely in the Fourier domain. We show that the polygon's Fourier descriptors may be obtained from the corresponding descriptors of the contour, and also through the DFT of the vertex locations. The results illuminate connections between sampling and interpolation in the Fourier domain.

\section{NOTATION AND CONVENTIONS}

Let $\mathcal{C}$ denote a closed, simple contour located in the complex plane $\mathbb{C}$. $\mathcal{C}$ may be parameterized by a complexvalued function $z$, with parameter $t \in[0,1]$. From standard differential geometry, the speed of the contour is $\left|z^{\prime}\right|$, and the arc length of a segment drawn by $t \in[0, A]$, with $0 \leq A \leq 1$ is

$$
s(A)=\int_{0}^{A}\left|z^{\prime}(t)\right| d t .
$$

Contact information

MA: E-mail: m.andrews@auckland.ac.nz

RK: E-mail: ramakrishna@ntu.edu.sg

Intelligent Robots and Computer Vision XXXII: Algorithms and Techniques, edited by Juha Röning, David Casasent

Proc. of SPIE-IS\&T Electronic Imaging, Vol. 9406, 94060D - (c) 2015 SPIE-IS\&T

CCC code: $0277-786 \mathrm{X} / 15 / \$ 18 \cdot$ doi: $10.1117 / 12.2078148$

Proc. of SPIE-IS\&T/ Vol. 9406 94060D-1 


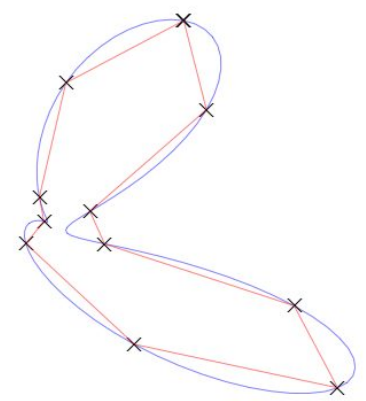

Figure 1. A contour $\mathcal{C}$ is sampled at $N=11$ points, indicated with "X", and the samples connected to form a polygonal approximation.

Let $L=s(1)$ denote the total arc length of the contour. Differential geometry ${ }^{6}$ shows that $\mathcal{C}$ may also be parameterized by a unit-speed function $w$ whose argument is arc-length $s$, with $0 \leq s \leq L$. Hence, both $z$ and $w$ parametrize the same contour, with varying and constant speeds, respectively.

Both $z$ and $w$ are periodic functions, since $\mathcal{C}$ is closed. Hence, they may be expanded in a Fourier series. Since the varying speed parameterization is more general of the two, we use it for analysis in this paper. Specifically, for $z$, this is

$$
z(u)=\sum_{k=-\infty}^{\infty} c_{k} e^{j 2 \pi k u}, \quad 0 \leq u \leq 1
$$

It should be understood that " $u$ " may represent any parameterization, including arc length.

Suppose that $\mathcal{C}$ is sampled at a set of $N$ points, denoted $p[0], p[1], \ldots, p[N-1]$. When connected together, as shown in Figure 2, the points form a polygon. Each vertex is a point in the complex plane, and the discrete Fourier transform (DFT) of the vertices is

$$
P[k]=\sum_{n=0}^{N-1} p[n] e^{-j 2 \pi k n / N} .
$$

The samples of any complex-valued function may be interpolated to form a continuous function. While the ideal interpolator for band limited samples is the sinc function, simpler interpolators may be obtained from the zero-order hold

$$
r_{0}(u)= \begin{cases}1, & \text { if }|u| \leq \frac{1}{2} \\ 0, & \text { otherwise }\end{cases}
$$

The first order hold interpolator, $r_{1}$, is obtained by convolving $r_{0}$ with itself: $r_{1}=r_{0} * r_{0}$, and is described as:

$$
r_{1}(u)= \begin{cases}1-|u|, & \text { if }|u| \leq 1 \\ 0, & \text { otherwise }\end{cases}
$$

The Fourier transform of $r_{0}$ is

$$
R_{0}(f)=\int_{-\infty}^{\infty} r_{0}(u) e^{-j 2 \pi f u} d u=\operatorname{sinc}(f) .
$$

Consequently, the first order hold has the transform

$$
R_{1}(f)=\operatorname{sinc}^{2}(f) .
$$




\section{POLYGONAL APPROXIMATION IN THE FOURIER DOMAIN}

The main results of our paper connect the Fourier series coefficients of a contour with those of a polygonal approximation obtained by connecting samples taken from the contour. Suppose a closed, simple contour is represented by a complex-valued function $x$, with argument $u \in[0,1]$ denoting an arbitrary parameter.

Write $x$ in a Fourier series as

$$
x(u)=\sum_{m=-\infty}^{\infty} b_{m} e^{j 2 \pi m u} .
$$

If we sample $x$ at $N$ evenly-spaced points, $u=0,1 / N, \ldots,(N-1) / N$, and connect those points with straight lines to form a polygon, then the polygon's contour may also be represented by a periodic complex-valued function $z$ with Fourier series

$$
z(t)=\sum_{k=-\infty}^{\infty} c_{k} e^{j 2 \pi k t}
$$

Our first result connects the polygon's Fourier coefficients with those of the underlying contour.

THEOREM 3.1. The Fourier coefficients of the polygon in (9) are obtained from those of the contour in (8) as follows:

$$
c_{k}=\operatorname{sinc}^{2}\left(\frac{k}{N}\right) \sum_{m=-\infty}^{\infty} b_{k-N m}
$$

Proof. Since $x$ is a periodic function, its samples may be described using a Dirac comb on the real line as:

$$
x_{s}(u)=x(u) \sum_{n=-\infty}^{\infty} \delta\left(u-\frac{n}{N}\right) .
$$

The sampling operation at spacing $1 / N$ transforms in the Fourier domain to another Dirac comb with reciprocal spacing. Consequently, the Fourier transform of (11) is,

$$
X_{s}(f)=N \sum_{n=-\infty}^{\infty} X(f-N n) .
$$

The continuous-time Fourier transform $X$ of the underlying curve with series (2) is

$$
X(f)=\sum_{m=-\infty}^{\infty} b_{m} \delta(f-m) .
$$

Putting (13) into (12) gives

$$
X_{s}(f)=N \sum_{n=-\infty}^{\infty} \sum_{m=-\infty}^{\infty} b_{m} \delta(f-N n-m) .
$$

Connecting the samples of $x_{s}$ with straight lines is equivalent to convolving (11) with the first order hold interpolation function $r_{1}(N u)$, where the scale factor $N$ applied to the argument to account for the $1 / N$ spacing of samples. The corresponding Fourier transform of the interpolator is

$$
\mathcal{F}\left\{r_{1}(N u)\right\}=\frac{1}{N} R_{1}\left(\frac{f}{N}\right)=\frac{1}{N} \operatorname{sinc}^{2}\left(\frac{f}{N}\right) .
$$

Consequently, the polygon obtained by $z(u)=x_{s}(u) * r_{1}(N u)$ has the transform:

$$
\begin{aligned}
Z(f) & =\sum_{n=-\infty}^{\infty} \sum_{m=-\infty}^{\infty} b_{m} \operatorname{sinc}^{2}\left(\frac{f}{N}\right) \delta(f-N n-m), \\
& =\sum_{n=-\infty}^{\infty} \sum_{m=-\infty}^{\infty} b_{m} \operatorname{sinc}^{2}\left(\frac{N n+m}{N}\right) \delta(f-N n-m)
\end{aligned}
$$


The inverse transform of $Z$ yields

$$
z(t)=\sum_{n=-\infty}^{\infty} \sum_{m=-\infty}^{\infty} b_{m} \operatorname{sinc}^{2}\left(\frac{N n+m}{N}\right) e^{j 2 \pi(N n+m) t}
$$

Grouping terms of $k=N n+m$ yields the desired result:

$$
z(t)=\sum_{k=-\infty}^{\infty} \operatorname{sinc}^{2}\left(\frac{k}{N}\right)\left[\sum_{n=-\infty}^{\infty} b_{k-N n}\right] e^{j 2 \pi k t}
$$

$\square$

The Theorem requires the Fourier series coefficients $b$ of the original curve, which may not always be known. It seems reasonable to expect to obtain the desired coefficients in (9) using only the vertices of the polygon formed by the samples. We now consider how this works.

As above, let $p[n], 0 \leq n \leq N-1$, denote the vertices of the polygon obtained by taking $N$ samples of the curve $x(u), 0 \leq u \leq 1$, i.e., $p[n]=x(n / N)$, with corresponding DFT (3). It is understood that $P[k+N]=P[k]$.. The polygon forms a closed curve, with Fourier series (9). We establish the following connection.

THEOREM 3.2. The Fourier series coefficients of the polygon (9) are obtained from the DFT of the vertices as follows:

$$
c_{k}=\frac{1}{N} P[k] \operatorname{sinc}^{2}\left(\frac{k}{N}\right) .
$$

With these coefficients, the curve (9) satisfies

$$
z(n / M)=p[n], \quad 0 \leq n \leq N-1 .
$$

Proof. The polygon is described by the following sequence of convolutions, which repeat one period over the real line followed by interpolation:

$$
z(t)=\left[\sum_{n=0}^{N-1} p[n] \delta(t-n / N)\right] *\left[\sum_{k=-\infty}^{\infty} \delta(t-k)\right] * r_{1}(N t) .
$$

In the Fourier domain, this results in two multiplications:

$$
Z(f)=\left[\sum_{n=0}^{N-1} p[n] e^{-j 2 \pi f n / N}\right]\left[\sum_{k=-\infty}^{\infty} \delta(f-k)\right]\left[\frac{1}{N} \operatorname{sinc}^{2}(f / N)\right] .
$$

Combining summations, and using the sampling property of the Dirac delta function, we obtain

$$
Z(f)=\frac{1}{N} \sum_{k=-\infty}^{\infty}\left[\sum_{n=0}^{N-1} p[n] e^{-j 2 \pi k n / N}\right] \operatorname{sinc}^{2}\left(\frac{k}{N}\right) \delta(f-k) .
$$

The sum in brackets is the DFT (3). We obtain (18) by taking the inverse Fourier transform:

$$
z(t)=\frac{1}{N} \sum_{k=-\infty}^{\infty} P[k] \operatorname{sinc}^{2}\left(\frac{k}{N}\right) e^{-j 2 \pi k t} .
$$


To prove (19), we see from (20) that

$$
\begin{aligned}
z(t) & =\left[\sum_{n=0}^{N-1} p[n] \delta(t-n / N)\right] * \sum_{k=-\infty}^{\infty} r_{1}(N t-N k) \\
& =\sum_{n=0}^{N-1} \sum_{k=-\infty}^{\infty} p[n] \int_{-\infty}^{\infty} \delta(\tau-n / N) r_{1}(N t-N \tau-N k) d \tau \\
& =\sum_{n=0}^{N-1} \sum_{k=-\infty}^{\infty} p[n] r_{1}(N t-n-N k)
\end{aligned}
$$

Substituting $t=m / N$, we have

$$
z(m / N)=\sum_{n=0}^{N-1} p[n] r_{1}(m-n-N k) .
$$

From (5), it follows that $r_{1}(m-n-N k)=0$, unless $m-n=N k$. For $0 \leq m, n<N$, this requires $k=0$, and $m=n$. Therefore, $z(m / N)=p[m]$, as desired.

The two Theorems lead to the following identity.

Corollary 3.3. For $N$ samples $p[n]=x(n / N), 0 \leq n<N$, from a curve $x$ with Fourier series (8), we have

$$
P[k]=N \sum_{m=-\infty}^{\infty} b_{k-N m}, \quad k \neq \pm N, \pm 2 N, \ldots
$$

Although the Corollary is a direct result of the theorems, insight comes from the following analysis. Substitution shows that

$$
\sum_{n=0}^{N-1} x(n / M) e^{-j 2 \pi k n / N}=N \sum_{m=-\infty}^{\infty} \int_{0}^{1} x(u) e^{-j 2 \pi(k-N m) u} d u .
$$

Simplifying, we find that the right hand side becomes

$$
N \int_{0}^{1} x(u) e^{-j 2 \pi k u}\left[\sum_{m=-\infty}^{\infty} e^{j 2 \pi N m u}\right] d u .
$$

The sum in brackets is reducible using the Poisson summation formula to

$$
\sum_{m=-\infty}^{\infty} e^{j 2 \pi N m u}=\sum_{n=-\infty}^{\infty} \delta(N u-n)=\frac{1}{N} \sum_{n=-\infty}^{\infty} \delta(u-n / N) .
$$

Substituting this back into (29) shows that both sides are indeed equal. Further insight into the two theorems comes from examining additional points.

1. The sinc function is zero at all integers except zero, and hence the polygon's Fourier descriptors $c_{k}$ vanish for $k= \pm N, \pm 2 N, \pm 3 N, \ldots$.

2. Combining (18) and (19), we obtain the interesting identity

$$
p[n]=\frac{1}{N} \sum_{k=-\infty}^{\infty} P[k] \operatorname{sinc}^{2}\left(\frac{k}{N}\right) e^{j 2 \pi k n / N} .
$$

This is an interesting variation on the inverse DFT formula:

$$
p[n]=\frac{1}{N} \sum_{k=0}^{N-1} P[k] e^{j 2 \pi k n / N} .
$$


The relationship between (32) and (33) is understood by substituting for the DFT in (32), yielding

$$
p[n]=\frac{1}{N} \sum_{k=-\infty}^{\infty}\left[\sum_{u=0}^{N-1} p[u] e^{-j 2 \pi u k / N}\right] \operatorname{sinc}^{2}\left(\frac{k}{N}\right) e^{j 2 \pi k n / N}
$$

Rearranging terms gives

$$
p[n]=\sum_{u=0}^{N-1} p[u]\left[\frac{1}{N} \sum_{k=-\infty}^{\infty} \operatorname{sinc}^{2}\left(\frac{k}{N}\right) e^{-j 2 \pi k(u-n) / N}\right] .
$$

Hence, we must have

$$
\frac{1}{N} \sum_{k=-\infty}^{\infty} \operatorname{sinc}^{2}\left(\frac{k}{N}\right) e^{-j 2 \pi k \ell / N}=\delta[\ell],
$$

where $\delta[\ell]$ is the Kronecker delta. In particular, setting $\ell=0$, we have

$$
\frac{1}{N} \sum_{k=-\infty}^{\infty} \operatorname{sinc}^{2}\left(\frac{k}{N}\right)=1
$$

Setting $N=1$ gives the obvious result:

$$
\sum_{k=-\infty}^{\infty} \operatorname{sinc}^{2}(k)=1
$$

Interestingly, it can be shown ${ }^{7}$ that:

$$
\sum_{k=-\infty}^{\infty} \frac{\sin (k)}{k}=\sum_{k=-\infty}^{\infty} \frac{\sin ^{2}(k)}{k^{2}}=\pi
$$

3. By inserting (18) into (2), and grouping terms involving each DFT coefficient, we obtain:

$$
z(t)=\frac{1}{N} \sum_{m=0}^{N-1} P[m] \sum_{k=-\infty}^{\infty} \operatorname{sinc}^{2}\left(\frac{m}{N}+k\right) e^{j 2 \pi(m+k N) t} .
$$

In particular, since $\operatorname{sinc}^{2}(k)=\delta[k]$, we have

$$
z(t)=\frac{P[0]}{N}+\frac{1}{N} \sum_{m=1}^{N-1} \sum_{k=-\infty}^{\infty} P[m] \operatorname{sinc}^{2}\left(\frac{m}{N}+k\right) e^{j 2 \pi(m+k N) t}
$$

This shows that the "center" of the polygon is at $1 / N \sum_{n=0}^{N-1} z[n]$. For $N=1$, the polygon is a single point $z[0]$, and we obtain

$$
z(t)=p[0]
$$

as expected. For $N=1$, the polygon is a line from $p[0]$ to $p[1]$, and back again. The curve then becomes

$$
z(t)=\frac{1}{2}\left[P[0]+P[1] \sum_{k=-\infty}^{\infty} \operatorname{sinc}^{2}\left(\frac{1}{N}+k\right) e^{j 2 \pi(2 k+1) t}\right] .
$$

Since $P[0]=p[0]+p[1]$ and $P[1]=p[0]-p[1]$, it follows that at $t=0$, we have $z(0)=1 / 2(P[0]+P[1])=$ $p[0]$, as expected. We must have $z(1 / 2)=p[1]$ by $(19)$. Hence, we obtain again another interesting identity:

$$
\sum_{k=-\infty}^{\infty} \operatorname{sinc}^{2}\left(\frac{1}{N}+k\right)=1
$$

Remarkably, this result is independent of $\mathrm{N}$. 


\section{INTERPOLATORS AND POLYGONS}

The analysis of the previous section relied on the first order hold and its transform, the sinc squared function. Other interpolators are, of course, possible and it is interesting to analyze their effect in the Fourier domain. As we have seen, the analysis may be carried out in the frequency domain, requiring only the transform of the interpolating function. For example, if the interpolator has transform $R(f)$, then Theorems (3.1) and (3.2) show that:

$$
\begin{aligned}
c_{k} & =N R\left(\frac{k}{N}\right) \sum_{n=-\infty}^{\infty} b_{k-N n}, \\
c_{k} & =P[k] R\left(\frac{k}{N}\right) .
\end{aligned}
$$

If, for example, the zero-order-hold (4) is used, the transform is $\frac{1}{N} \operatorname{sinc}(f / N)$, and the resulting expressions are:

$$
\begin{aligned}
c_{k} & =\operatorname{sinc}\left(\frac{k}{N}\right) \sum_{n=-\infty}^{\infty} b_{k-N n}, \\
c_{k} & =\frac{1}{N} P[k] \operatorname{sinc}\left(\frac{k}{N}\right) .
\end{aligned}
$$

The ideal interpolator, with samples spaced $1 / N$ apart, is the sinc function:

$$
r_{i}(u)=\operatorname{sinc}(N u)
$$

with corresponding transform

$$
R_{i}(f)= \begin{cases}1 / N, & \text { if }|f| \leq \frac{N}{2} \\ 0, & \text { otherwise }\end{cases}
$$

Using the ideal interpolator, we have

$$
\begin{aligned}
c_{k} & =R_{i}\left(\frac{k}{N}\right) \sum_{n=-\infty}^{\infty} b_{k-N n}, \\
c_{k} & =\frac{1}{N} P[k] R_{i}\left(\frac{k}{N}\right) .
\end{aligned}
$$

It is not obvious that this recovers the original samples, as it should. The Appendix shows that by revisiting the proof of Theorem 3.1, we find perfect recovery.

An interesting interpolator that preserves the original samples is the cardinal spline of the 2 -nd order, ${ }^{8}$ which has the transform

$$
R_{2}^{c}(f)=\frac{4 \operatorname{sinc}^{3}(f)}{3+\cos (2 \pi f)} .
$$

\section{SUMMARY AND CONCLUSIONS}

Simplifying shapes into polygons is a basic operation in computer graphics and computer vision. This paper derives mathematical results connecting the Fourier descriptors of the underlying shape to those of the polygon. The Fourier descriptors are shown to be obtained from the vertices using the DFT, and also by summing groups of coefficients of the underlying curve. The results extend to cover other interpolators.

The relationships derived in this paper illuminate connections between sampling theory and the planar geometry of curves and polygon that are not well known. It is interesting that such results are obtained in the Fourier domain. In future, we explore whether the Fourier series basis functions are indeed the best ones when representing polygons. 


\section{Appendix}

We show that the ideal interpolator recovers samples exactly. Given that the sampled function, repeated across the real line, has transform

$$
X(f)=\sum_{n=0}^{N-1} \sum_{k=-\infty}^{\infty} p[n] e^{-j 2 \pi f n / N} \delta(f-k) .
$$

We obtain the interpolated signal $z_{i}$ by convolution with the ideal interpolator:

$$
z_{i}(t)=x(t) * r_{i}(t) .
$$

In the frequency domain, this is $Z_{i}(f)=X(f) R_{i}(f)$. This gives

$$
\begin{aligned}
Z_{i}(f) & =\sum_{n=0}^{N-1} \sum_{k=-\infty}^{\infty} p[n] R_{i}(f) e^{-j 2 \pi f n / N} \delta(f-k), \\
& =\frac{1}{N} \sum_{n=0}^{N-1} p[n]\left[\sum_{k=-\lfloor N / 2\rfloor+1}^{\lfloor N / 2\rfloor} e^{-j 2 \pi f n / N} \delta(f-k)\right] .
\end{aligned}
$$

The sum in brackets may be reorganized by noting that the exponentials $e^{-j 2 \pi f n / N}$ are periodic with period $N$, allowing us to write

$$
Z_{i}(f)=\frac{1}{N} \sum_{n=0}^{N-1} p[n] \sum_{k=-0}^{N-1} e^{-j 2 \pi f n / N} \delta(f-k)
$$

Applying the inverse Fourier transform to $Z_{i}$ gives us

$$
\begin{aligned}
z_{i}(t) & =\int_{-\infty}^{\infty} Z_{i}(f) e^{-j 2 \pi f t} d t \\
& =\frac{1}{N} \sum_{n=0}^{N-1} p[n] \sum_{k=0}^{N-1} \int_{-\infty}^{\infty} e^{-j 2 \pi f n / N} e^{j 2 \pi f t} \delta(f-k) d t \\
& =\frac{1}{N} \sum_{n=0}^{N-1} p[n] \sum_{k=0}^{N-1} e^{-j 2 \pi k n / N} e^{j 2 \pi k t} .
\end{aligned}
$$

Rearranging the sum gives

$$
z_{i}(t)=\frac{1}{N} \sum_{k=0}^{N-1}\left[\sum_{n=0}^{N-1} p[n] e^{-j 2 \pi k n / N}\right] e^{j 2 \pi k t} .
$$

The sum in brackets is simply the DFT of the polygon vertices, yielding

$$
z_{i}(t)=\frac{1}{N} \sum_{k=0}^{N-1} P[k] e^{j 2 \pi k t} .
$$

In particular, we have, by the inverse DFT, that

$$
z_{i}(n / N)=\frac{1}{N} \sum_{k=0}^{N-1} Z[k] e^{j 2 \pi k n / N}=z[n] .
$$

\section{ACKNOWLEDGMENTS}

The support of the Singapore Ministry of Education under grant MOE-T2-1-010 is gratefully acknowledged. 


\section{REFERENCES}

[1] Ling, H. and Jacobs, D., "Inner distance using the shape context," Pattern Analysis and Machine Intelligence, IEEE Transactions on 29, 286-289 (2007).

[2] Premachandran, V. and Kakarala, R., "Perceptually motivated shape context which uses shape interiors," Pattern Recognition 46, 2092-2102 (2013).

[3] Zahn, C. T. and Roskies, R. Z., "Fourier descriptors for plane closed curves," Computers, IEEE Transactions on C-21, 269-281 (March 1972).

[4] Douglas, D. and Peucker, T., "Algorithms for the reduction of the number of points required to represent a digitized line or its caricature," The Canadian Cartographer 10(3), 112-122 (1973).

[5] Osada, R., Funkhouser, T. A., Chazelle, B., and Dobkin, D. P., "Shape distributions," ACM Trans. Graph. 21(4), 807-832 (2002).

[6] do Carmo, M. P., [Differential Geometry of Curves and Surfaces], Prentice-Hall, Upper Saddle River, NJ (1976).

[7] Baillie, R., Borwein, D., and Borwein, J. M., "Surprising sinc sums and formulas," The American Mathematical Monthly 115(10), 888-901 (2008).

[8] Aldroubi, A., Unser, M., and Eden, M., "Cardinal spline filters: Stability and convergence to the ideal sinc interpolator," Signal Processing 28, 127-138 (Aug. 1992). 